\title{
Miniaturized drug delivery system TUDOS with accurate metering of microliter volumes
}

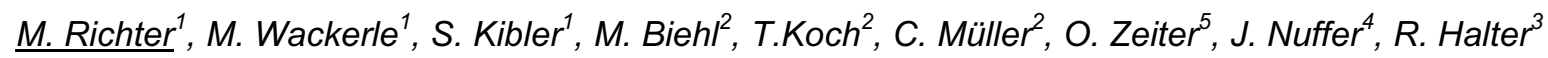 \\ ${ }^{1}$ Fraunhofer Institution for Modular Solid State Technologies EMFT \\ ${ }^{2}$ Fraunhofer Institute for Biomedical Engineering IBMT \\ ${ }^{3}$ Fraunhofer Institute for Toxicology and Experimental Medicine ITEM \\ ${ }^{4}$ Fraunhofer Institute for Structural Durability and System Reliability LBF \\ ${ }^{5}$ Fraunhofer Institute for Reliability and Microintegration IZM
}

\begin{abstract}
A miniaturized drug delivery system for accurate dosing of drugs for therapy of cancer is presented. The weight of the system is less than 8 grams. Silicon micropumps with a stroke volume of $88 \mathrm{nl}$, and blocking pressure of 6 bar, drive the system. A novel and accurate flow monitoring principle based on capacitance measurement was developed and implemented into the drug delivery system. To prevent free flow, a self blocking safety valve was adapted to the drug micropump. Moreover, further safety features like catheter blockage detection, pump failure detection, bubble detection and a bubble separator was realized. System control and driver electronics to supply the pumps with high voltage was realized on a tiny flex PCB and integrated. System tests showed, that test dosing volumes of $12 \mu \mathrm{l}$ can be delivered reliably with accuracy better than $4 \%$. The system is now ready for animal trial (rat model) for a new cancer therapy approach.
\end{abstract}

Key words: Microdosing, Drug delivery system, Micropump, Tumor therapy, Flow control

\section{Introduction}

There are two classes of miniaturised drug delivery systems: Highly miniaturised but not controllable steady flow osmotic pumps (class 1) and controllable systems based on conventional pumps or micro pumps (class 2)

Extremely miniaturized pump systems are based on the osmotic pump principle [1] (class 1). The driving force is given by a concentration gradient. Before operation, these pump units are loaded with a drug, there is a steady drug flow at a given flow rate. T. Velten et. al. integrated an osmotic pump with the size of two teeth inside the human mouth [2]. From Alzet [3] miniaturised pump systems are available for animal trial in mice, the weight of the pump systems is varying between $0.4 \mathrm{~g}$ and $6 \mathrm{~g}$.

Goldschmidtböing et. al. published a drug delivery system for cancer therapy [4]. This approach is based on two piezoelectric diaphragms, one of which is acting as an active valve. As a drawback, a bubble tolerance of this pump was not shown for the relevant operation conditions, and still the overall weight of the system amount to $25 \mathrm{~g}$, which is relatively high.

Concerning drug delivery systems with controllable pumps or micro pumps, a miniaturised drug delivery system is commercially available for subcutaneous implantation into lab animals as small as rats (weight $10 \mathrm{~g}$ ). [5]

Leading edge of class 2 can be considered the pump system from iPRECIO [6]. The range of flow rate is between $1.0 \mu \mathrm{L} / \mathrm{hr}-30.0 \mu \mathrm{L} / \mathrm{hr}$ (Resolution : $0.1 \mu \mathrm{L}$ ) with an accuracy of better $\pm 5 \%$, with a size of $38.7 \times 19.2 \times 9.7 \mathrm{~mm}^{3}$ and $a$ weight of 7.9 grams. This system can be considered as a benchmark within class 2, also for the drug delivery system (TUDOS) which is presented in this study.

Class 1 can be miniaturised to a weight level $(0.4 \mathrm{~g})$ which is not possible for controllable pumps of class 2. As main drawback, the flow of class 1 can not be varied. Within class 2 the best conventional pumps is even smaller $(7.9 \mathrm{~g})$ compared to the micropump system $(25 \mathrm{~g})$.

Moreover, neither class 1 nor class 2 pump systems, which are miniaturised below $10 \mathrm{~g}$, have neither flow measurement features nor stall pressure larger $100 \mathrm{kPa}$, bubble separator, and safety features like bubble detection and pump failure detection.

Here, the target of the fully programmable TUDOS system (class 2), will be on the one hand the miniaturisation to a weight of less than 7.5 $\mathrm{g}$, which would be comparable to the leading edge of class $2(7.9 \mathrm{~g})$, and on the other hand include all the features mentioned above, which 
are not available in state-of-the-art systems today.

\section{Desired medical application}

The accurate dosing of very small quantities of liquids or gases is a major challenge in microfluidics. For drug delivery systems, e.g. for pain therapy, therapy of diabetes, hormone therapy or cancer therapy, there is a huge demand for accurate dosing of small amount of drugs.

In the final phase of a cancer disease the tumor is too big to be treated by surgery or chemotherapy. The TUDOS approach is to deliver a very small amount of highly concentrated cytostatics not into the circulatory system, but directly into the tumor for shrinking it. Therefore we started to develop a cancer model in rats. Two cancer models in rats were selected and will be tested for applicability. The duration of the treatment is planned to be 2 weeks, the TUDOS system shall work with one battery charge for four weeks.

\section{Concept of the demonstrator}

State of the art micro pumps are not accurate enough, due to influences like gas bubbles remaining in the pump chamber, back pressure, manufacturing tolerance, etc. With the said, a method to control the flow accurately is needed. The core of the concept is to combine two (possibly inaccurate and bubble dependent) micropumps with one (ore more) capacitive flow sensing unit and a bubble separator in a closed loop control (fig. 1) to achieve highly accurate dosing results.

\section{Novel flow metering principle}

The air pump is injecting an air bubble into the drug flow. The air bubble is acting like a piston, moving the drug in front of the air bubble towards the patient. The position of the interface air/drug can be detected accurately by measuring the capacitance of the capillary forming a meander. Between the electrodes of the meas- urement capacitance there is either drug or air. The difference of the dielectric coefficient between drug and air results in a measurement signal which is proportional to the position of the meniscus in the meander.

A volume of 12 micro liter can be metered with an accuracy of $4 \%$ and delivered to the animal. The injected air bubbles are removed from the drug stream by a bubble separator behind the sensor unit.

\section{Working principle}

First of all, the TUDOS system has to be calibrated. If the TUDOS system is dry after assembly, all five capacitances are measured, and the values are stored into the memory of the system.

The second calibration step is to prime the fluid path with drug by the drug pump, until the drug reaches the outlet. The capacitance values at the electrodes are monitored. If the final capacitance values for the four electrodes are stable, the mean value will be stored into the memory as the second value. After this calibration procedure, the capacitances for the full and empty micro channel are known. The calibration value for the safety electrode of the air pump will be derived from the value of the safety electrode of the drug pump.

\section{Dosing step of the TUDOS System}

The volume inside the channels between the Tjunction of air pump and drug pump and the stop electrode is defining the dosing volume $V_{D}$ of one dosing step. It amounts to $V_{D}=12 \mu \mathrm{l}$. The stroke volume $\Delta \mathrm{V}$ of the air pump is in the order of magnitude of $\Delta \mathrm{V}=83 \mathrm{nl}$ (it can slightly vary with back pressure). With that, it takes about $\mathrm{N}=\mathrm{V}_{\mathrm{D}} / \Delta \mathrm{V}=144$ pump strokes to perform the TUDOS dosing step. With an operation frequency of the air pump of $4 \mathrm{~Hz}$ this dosing step takes approx. 36 seconds.

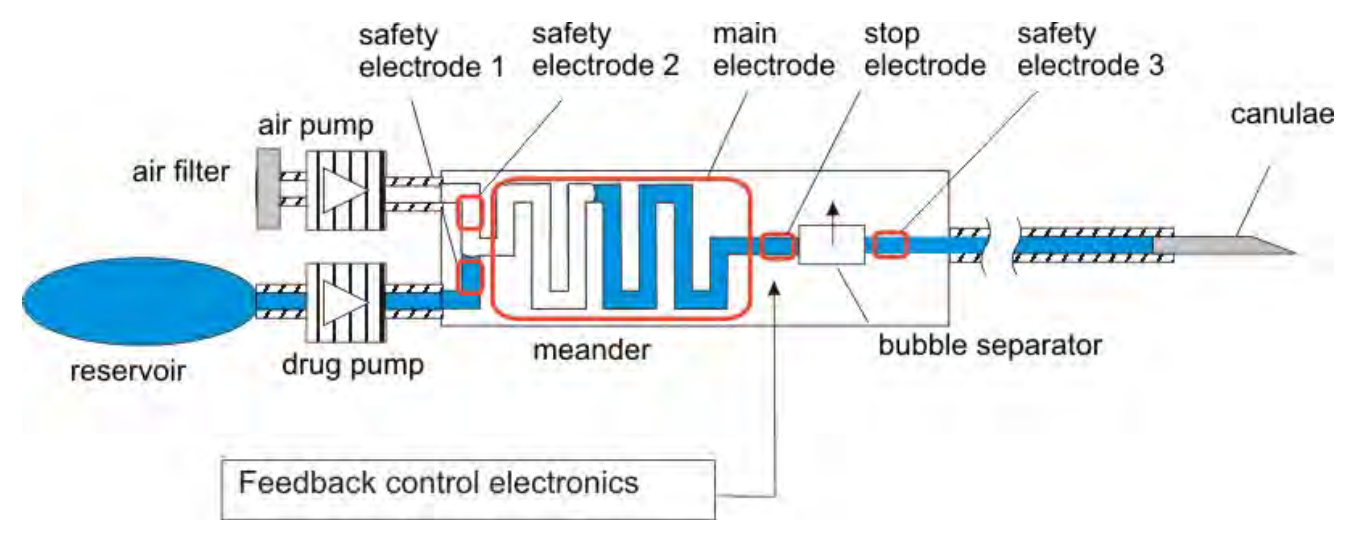

Fig. 1. Concept of the TUDOS drug dosing system 
In the mostly simple form, dosing can be performed in the following way:

1) air pump is doing one pump stroke

2) stop electrode will be checked: switch from 1 (drug) to 0 (air)?

- $Y$ : dosing mode is completed.

- $\quad \mathrm{N}$ : go back to step 1 to perform next pump stroke with the air pump)

With that, this procedure is very simple and therefore robust against software errors.

\section{Refill step of the TUDOS System}

The refill mode, executed by the drug pump, follows immediately after the dosing step in order to refill the dosing volume $V_{D}$ with drug again. The algorithm of this mode is like follows:

1) drug pump is doing one pump stroke

2) stop electrode will be checked: switch from 0 (air) to 1 (drug)?

- Y: dosing mode is completed.

- $\quad \mathrm{N}$ : go back to step 1 to perform next pump stroke with the drug pump)

\section{Safety checks}

To include safety checks, all electrodes can be read out after every pump stroke

1) Air pump is doing one pump stroke

2) Safety electrode after bubble separator will be read out: ERROR (Y/N)

3) Safety electrode of drug pump will be read out: ERROR (Y/N)

4) Safety electrode of air pump will be read out: $\operatorname{ERROR}(\mathrm{Y} / \mathrm{N})$

5) Large electrode will be checked: dosed volume has changed according to one pump stroke volume $\Delta \mathrm{V}(\mathrm{Y} / \mathrm{N})$ :

- $\mathrm{Y}$ : OK

- $\quad \mathrm{N}$ : ERROR (pump failure or blocked catheter)

Further safety features are implemented: pump failures or degradation of the pump valves, as well as catheter blockage, bubbles in the drug reservoir, and a failure of the bubble separator can be detected by safety electrodes.

\section{Silicon micropumps}

The piezoelectrically driven liquid and air micropumps (chip size $7 \times 7 \times 1 \mathrm{~mm}^{3}$, fig. 2) are based on silicon micro-machining. The compression ratio (ratio between stroke volume and dead volume) has been maximized, the pumps can achieve a very high back stall pressure (drug pump: 6 bar, air pump: 1 bar), which is strong enough to purge the catheter if it is blocked.

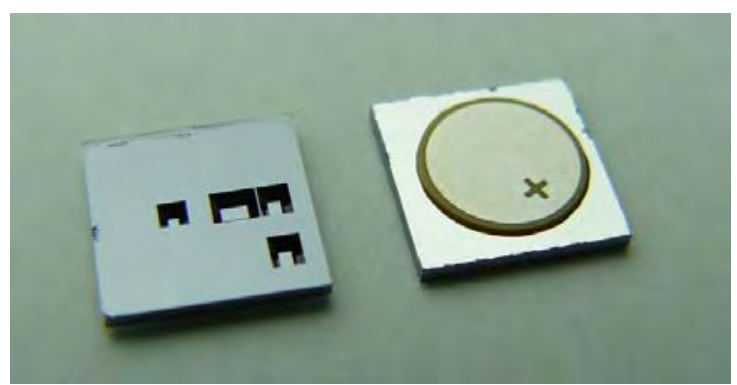

Fig.2. Silicon high pressure micropump with safety valve structure (left: bottom view with the fluidic ports, right: top view with round piezoceramic disc).

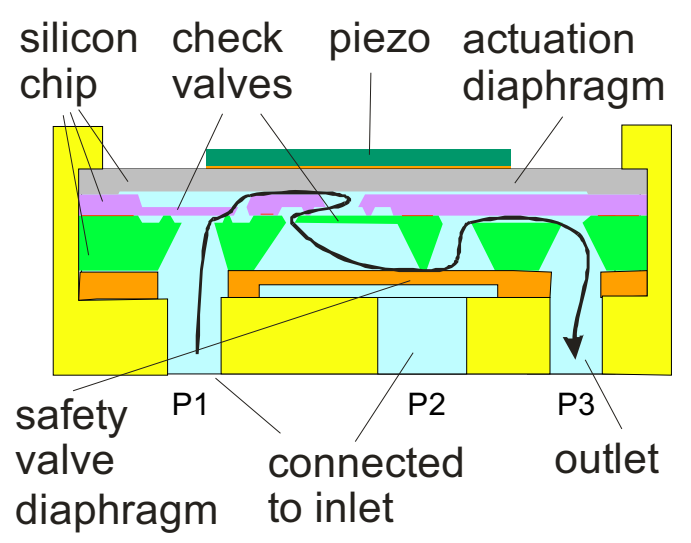

Fig. 3. Concept of the safety valve: the inlet pressure has a closing function (normally closed, self blocking) to the silicone safety valve diaphragm. The safety valve is opened if the pump is actuated by the overpressure generated by the pump stroke.

\section{Safety valve}

A special safety valve has been developed for the drug pump to avoid free flow, e.g. if the drug reservoir is pressurized by the animal or the patient (fig. 3). This safety valve is a silicone membrane, which at the same time serves as the gasket to seal the silicon pump chip to the fluidic module (fig. 4).

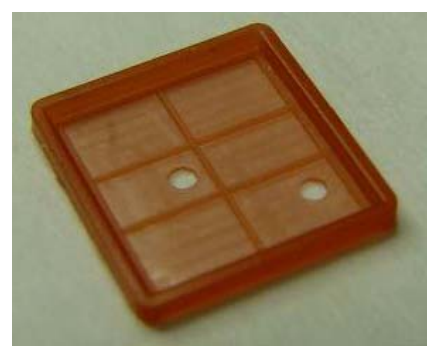

Fig. 4. Silicone gasket according to fig. 2, which is adapted to the bottom side of the silicon micropump (see fig. 2, left) 


\section{Fluidic module}

The micropumps and the safety valve are clamped to a fluidic module made of Poly Ether Ether Ketone (PEEK) (fig. 6). The fluidic channels are realized by milling. The channels are covered by a PEEK foil joined by laser welding (fig. 6).

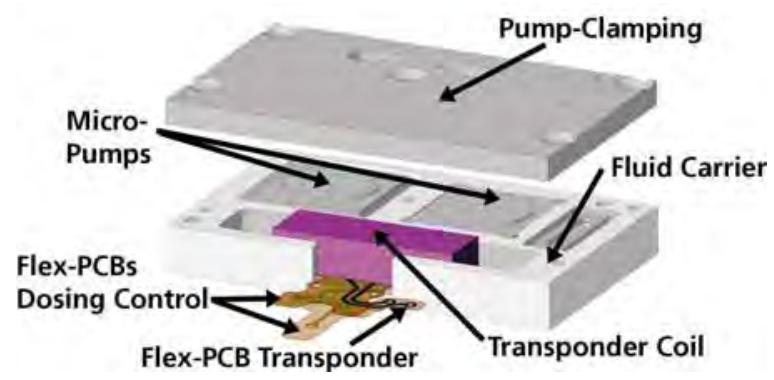

Fig. 5. Fluidic module made of PEEK, with micropumps, safety valve, bubble separator, electrodes and transponder coil

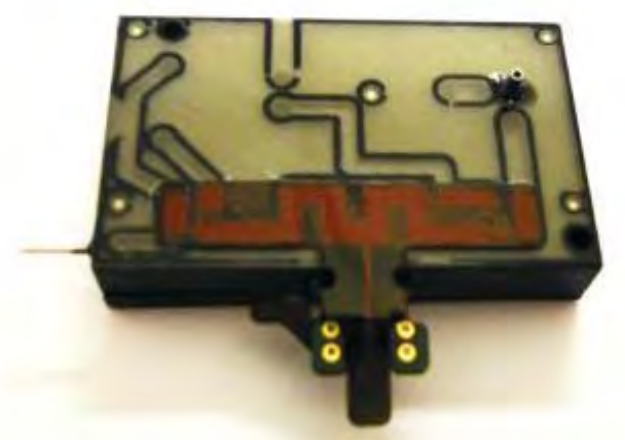

Fig. 6. Assembled fluidic module with mounted electrodes. The milled channels in the PEEK body are covered by a PEEK foil joined by laser welding, the black lines show the laser welding lines.

\section{System control and system development}

System- and driver-electronics have been arranged on a customized flexible printed circuit board (PCB) with a maximum thickness of $300 \mu \mathrm{m}$ (fig. 8). Even though connected, the high-voltage driver electronics and the system electronics are assembled spatially separated in order to avoid interference between high voltage and signal lines. The PCB provides electrical connections to dosing control electronics, transponder coil, both micro-pumps and battery. Two metallic contacts, which pass housing, allow for recharging of the battery. Using 3D-CAD, we could achieve sophisticated PCB-design and arrangement of components. The design realizes both, high area density with short connections between associated components thus minimizing cross-over, and highly compact 3D-packaging within the available housing cavity after the PCB has been folded (fig. 7).

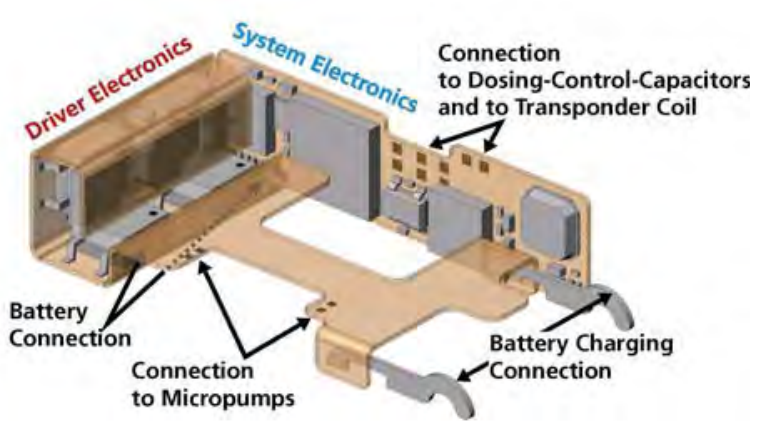

Fig. 7. Flexible PCB comprising pump driver electronics and system electronics in folded condition.

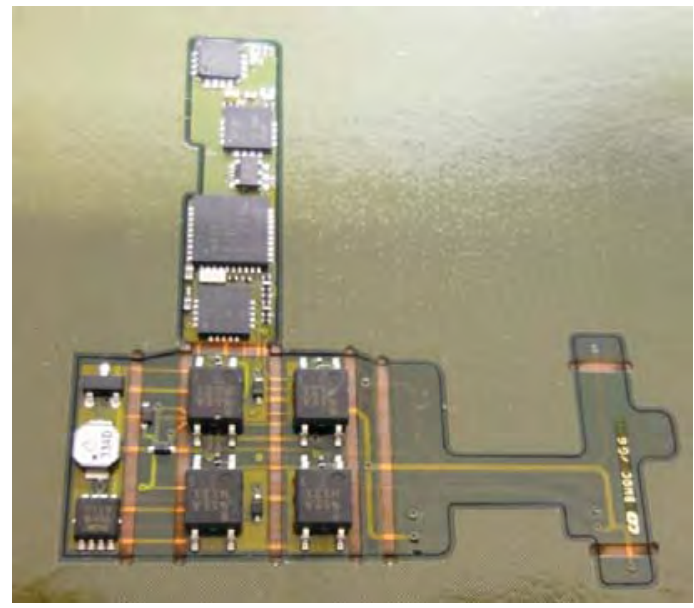

Fig. 8. Flex PCB with 6 PCB layer, not yet folded

The dosing profile can be sent to a microcontroller by a wireless user interface, the dosed drug volume is monitored and can be changed by the operator. For all components an interior space of less than $15 \mathrm{~mm} \times 16.5 \mathrm{~mm} \times 30 \mathrm{~mm}$ had been specified. A stable housing was produced by $3 \mathrm{D}$ printing to adapt the entire system to an animal trial with rats. Further, a miniaturized silicone drug reservoir was developed and adapted to the fluidic module.

Reservoir, fluid module and battery are stacked, with the $3 \mathrm{ml}$-reservoir on top claiming the largest volume (fig. 9 and 10).

The system- and driver-electronics is folded around fluid module and battery in the bottom compartment of the housing. The latter is separated from the top part containing the reservoir to avoid moisture contamination of electronics.

\section{Connection of Dosing-Control and Trans- ponder}

The dosing-control of the TUDOS-system is based on the capacitive detection of air bubbles, which are injected into the microfluidic channels of the fluid module. 


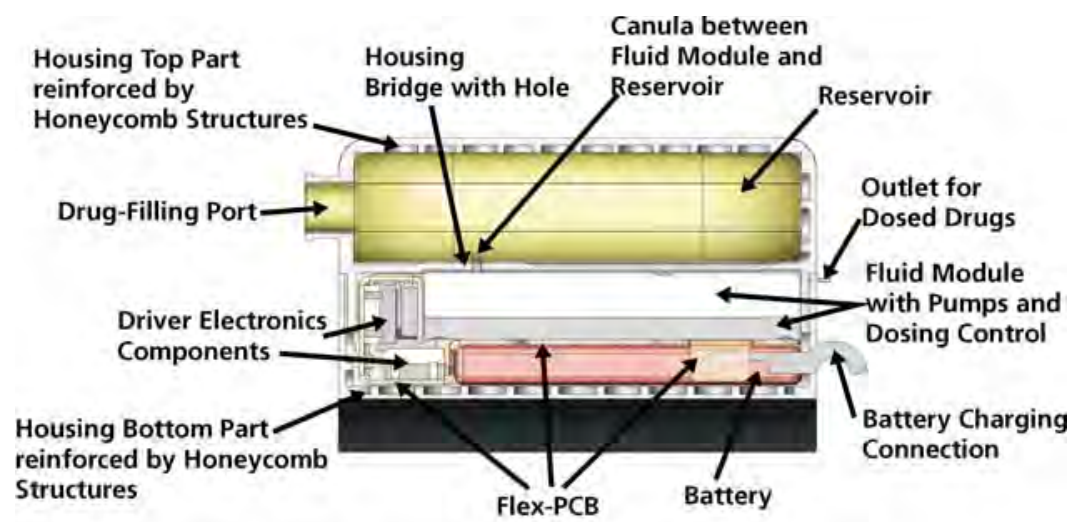

Fig. 9: Longitudinal section through the rat housing of the TUDOS drug dosing system. It reveals the arrangement of components within the housing.

The plates of the necessary plate-capacitors are fixed on two flexible PCBs, which must be positioned exactly opposite to each other with the related fluidic micro-channel in between.

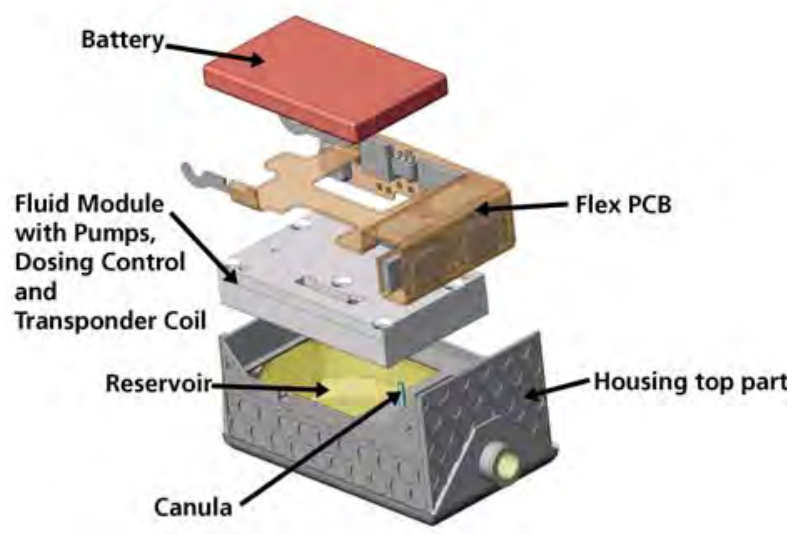

Fig. 10: Assembly of the TUDOS drug dosing system. All accommodated components are arranged within the top-part of the housing

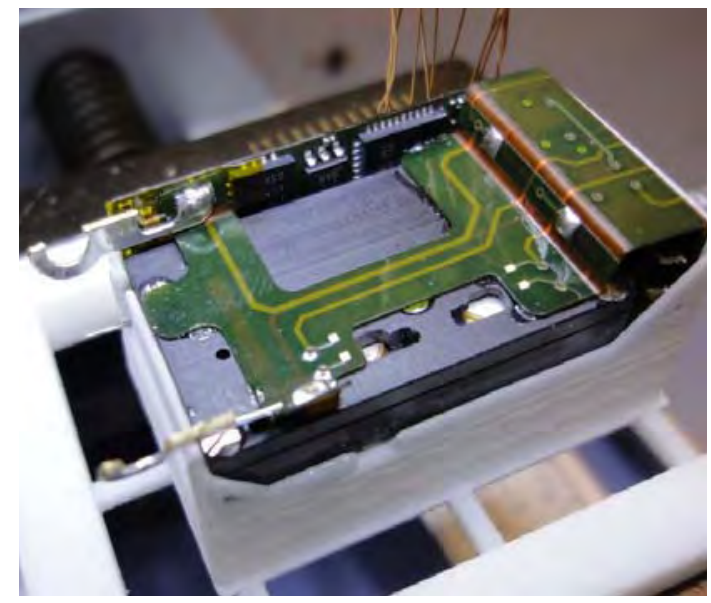

Fig. 11. Partly assembled TUDOS system.

In practice, one of these flex-PCBs is positioned outside the fluid module over the microchannels, the other one is positioned within the large cavity of the fluid module under the microchannels. This inner PCB is enveloped with another flex-PCB which is connected to the transponder-coil. After adjustment and conglutination of these flex-PCBs, their solder terminals are joined to the matched solder terminals of the folded system- and driver-PCB.

\section{Compatibility to drugs}

Cytostatics are very corrosive. For that, all materials to be in contact with cytostatics (PEEK, silicon, silicone) drugs have been tested to corrosion and lifetime.

\section{Fluidic tests of the micro pumps}

The back pressure performance was tested with water at a pump operation frequency of 5 $\mathrm{Hz}$ (fig 12). A stroke volume of $88 \mathrm{nl}$ was measured without back pressure. At a back pressure of $150 \mathrm{kPa}$ the reduction of the flow rate is about $15 \%$ compared to the value without back pressure. Above $150 \mathrm{kPa}$ the silicone gasket was not tight any longer, but from fig 12 an extrapolated blocking pressure of $600 \mathrm{kPa}$ can be derived. With that, this pump is strong enough to purge a blocked catheter easily.

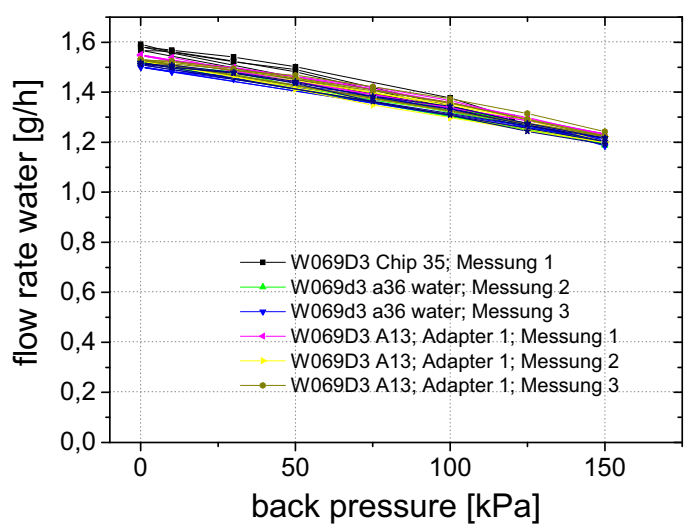

Fig. 12: Test of the silicon micropump with water. 


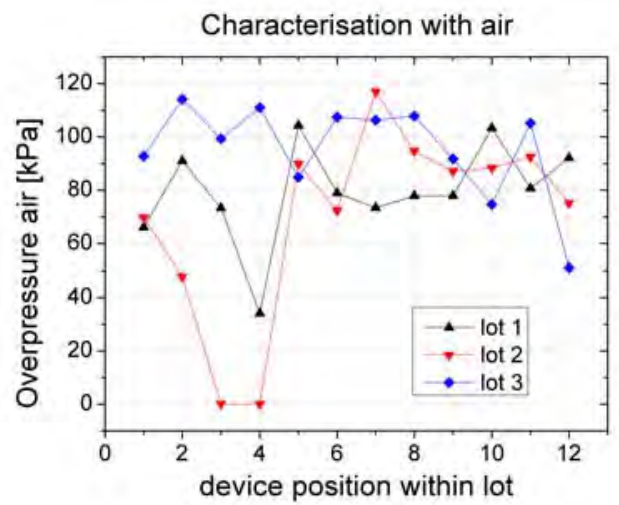

Fig. 13: Test of the silicon micropump with air. The lot (12 micro pumps each lot) were tested to air back pressure. Average back pressure ability with air is about $90 \mathrm{kPa}$.

Furthermore, also the air micropump must also show a high back pressure ability to perform the dosing step. Three lots (every lot 12 micropumps) have been assembled and tested (fig 13). A back pressure ability (with an operation frequency of $500 \mathrm{~Hz}$ ) of about 80 to $100 \mathrm{kPa}$ was achieved (fig 13), which is a factor of 3 greater compared to other known micro diaphragm pumps. With that high back pressure ability, the air pump can push forward the air meniscus during the dosing step even if a back pressure of about $80 \mathrm{kPa}$ is applied.

\section{Test of TUDOS system and dosing principle}

One fluid module was assembled and connected to a system electronics.

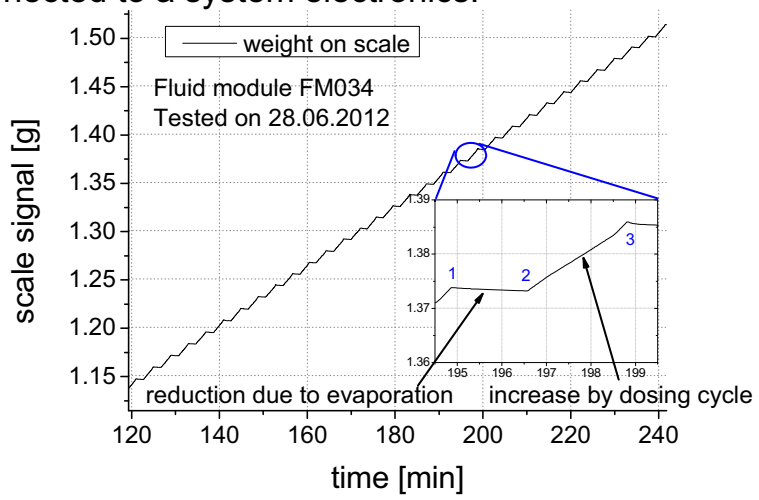

Fig. 14: Fluidic test (medium: water) of TUDOS drug dosing cycles using a scale

The system electronic was changing between activating the fluid pump and the air pump whenever the stop electrode changed its status between high capacitance (channel filled with water) and low capacitance (channel filled with air). Therefore a continuous delivery of the control volume of fluid (here water) was performed (fig. 14). For measuring the amount of delivered water for each dosing cycle the outlet of the fluid module was connected to a reservoir on a micro scale (SC2 from Satorius with a resolution of $0.1 \mu \mathrm{g}$ ).

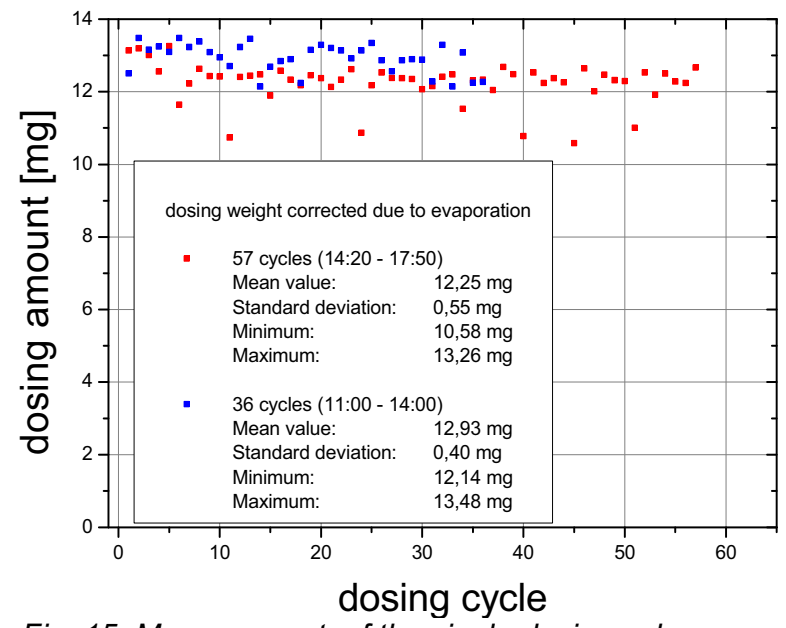

Fig. 15: Measurement of the single dosing volume

The assembled system have been tested, a dosing accuracy of $4 \%$ (standard deviation) have been shown. The gravimetrically measured volume value (fig 15) is in very good agreement with the geometric dosing volume $V_{D}$ of 12,3 $\mu \mathrm{l}$ between air/drug junction and stop electrode (compare fig. 1). With that, an accurate and back pressure independent metering principle of microliter volumes has been verified.

\section{Outlook}

The dosing principle of the TUDOS system has been tested successfully. Silicon micropumps with very high pressure abilities both for liquids and air have been realized. Currently, several TUDOS systems are assembled in order to start the animal trials with a rat cancer model.

\section{References}

[1] F. Theeuwes and S.I. Yum, Principles of the Design and Operation of Generic Osmotic Pumps for the Delivery of Semisolid or Liquid Drug Formulations, Alza research Palo Alto, USA, 1976)

[2] Drug Delivery Micro System for the Oral Cavity, Workshop Microdosing Systems, Fraunhofer IZM-M, Munich, 28.10.2008

[3] www.alzet.com (visited at 31.1.2013)

[4] F. Goldschmidtböing, A. Geipel, C. Farhat, P. Jantscheff, U. Massing, P. Woias, A miniaturized pressure independent drug delivery system for metronomic cancer therapy, 4th European Congress for Medical and Biomedical Engineering 2008, Engineering for Health, Antwerpen, 2008

[5] http://www.alnmag.com/article/infusion-pumpssmall-laboratory-animals?page $=0,1$ (visited at 31.1.2013)

[6] http://www.iprecio.com/products/specifications.ht $\underline{\mathrm{ml}}$. (visited at 31.1.2013) 